\title{
ASCA OBSERVATIONS OF CLUSTERS OF GALAXIES
}

\author{
KOUJUN YAMASHITA \\ Department of Physics, Nagoya University, Furo-cho, Chikusa, \\ Nagoya 464-01 JAPAN
}

\section{Introduction}

$\mathrm{X}$-ray emissions from clusters are most likely originated from a thin hot plasma in a collisional ionization equilibrium. The optical depth of continuum component is order of $10^{-3}$, whereas that of emission lines is around unity. Present emission models used for spectral fitting can not estimate this effect, so that the determination of elemental abundances seems to include large uncertainty. The high resolution spectroscopy with ASCA gives a clue to investigate the physical state of hot intracluster gas and a impact to reconsider the basic atomic processes. This is important issue to deeply understand the structure, formation and evolution of clusters, and the origin of intracluster gas.

The X-ray morphologies are divided into two categories, they are, centrally concentrated and spherically symmetric clusters with small core radii and cooling flows, and largely extended clusters with large core radii and merging substructures. ROSAT all sky survey detected thousands of clusters with the high angular resolution[1]. ASCA makes it possible to derive physical parameters of these clusters from the spatially-resolved spectroscopic observations[2]. The Hubble constant $\left(\mathrm{H}_{0}\right)$ was derived by observations of Sunyaev-Zeldovich (hereafter S-Z) effect clusters and by means of resonant scattering effect of emission lines in an intracluster gas.

Here we present the observational results of ASCA and discuss the cooling flow and merging of clusters, and the Hubble constant determination.

\section{Observations}

ASCA has already observed nearly 150 clusters of galaxies in the energy range of $0.5-10 \mathrm{keV}$ and in the redshift range up to 0.5 , including group of galaxies, poor clusters, nearby rich clusters $(z<0.1)$, distant clusters $(z>0.1)$ 
and superclusters. Some of S-Z effect clusters and gravitational lensing clusters were also observed. ASCA puts on board four sets of multi-nested thin foil conical mirror X-ray telescope (XRT) incorporated with two Xray $\mathrm{CCDs}$ (SIS) and imaging gas scintillation proportional counters(GIS) for each two sets[3]. The angular resolution and field of view are 3' (HPD), 22'x22' for SIS and 50'(diameter) for GIS, respectively. The energy resolutions are $2 \%$ and $8 \%$ at $6 \mathrm{keV}$ for SIS and GIS, respectively. ASCA instrumentations are described in detail by Serlemitsos et al.[4].

\section{Data processing}

The spectral analysis of observed data can be done by fitting a model spectra of thermal emissions from a thin hot plasma like Raymond-Smith or MEKA model, taking into account the absorption of neutral hydrogen in the line of sight. Thus the plasma temperature(kT) and abundance of each cluster corrected for the cosmological redshift $(z)$ were derived as shown in Fig.1, where 43 clusters are plotted. It is also important to fit a model of thermal bremsstrahlung and Gaussian lines as free parameters of kT, line intensities and energies. In this case we can derive an electron temperature from the continuum component, an ion temperature from intensity ratio of $\mathrm{H}$-like and He-like ions, abundance from line intensties of each element and the redshift from line energies. This procedure is very useful to qualify observed data. SIS has a capability to resolve emission lines of $\mathrm{H}$-like and He-like ions of heavy elements whereas GIS can distinguish $K_{\alpha}$ and $K_{\beta}$. Furthermore the optical depth of each line in an intracluster medium can be estimated from the intensity ratio of $\mathrm{K}_{\alpha}$ and $\mathrm{K}_{\beta}$. In Fig.2 there is shown an X-ray spectrum of $\mathrm{M} 87(\mathrm{z}=0.0038)$ within the radius of 2.5 ' observed by SIS and fitted with a thermal bremsstrahlung of $\mathrm{kT}=1.9 \mathrm{keV}$. Residuals clearly show the existence of several emission lines, such as $\mathrm{O}, \mathrm{Si}, \mathrm{S}, \mathrm{Ar}, \mathrm{Ca}$ and Fe.

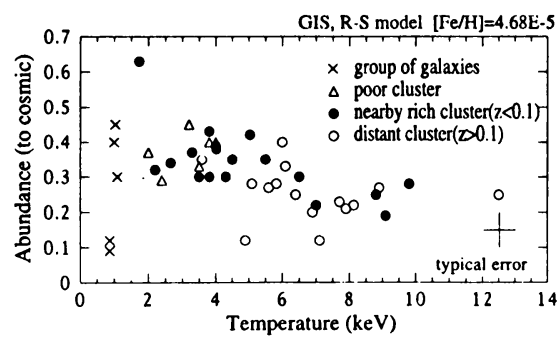

Figure 1. Abundance vs. plasma temperature for 43 clusters

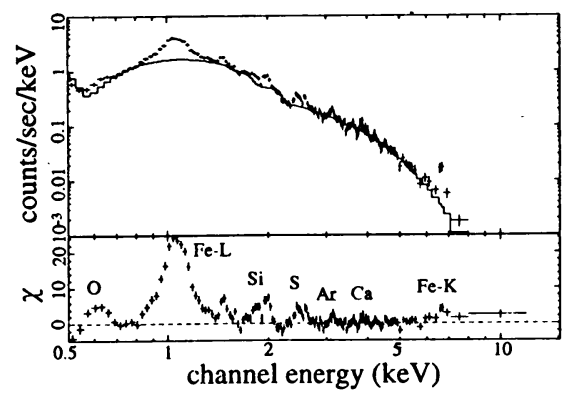

Figure 2. X-ray spectrum of $\mathrm{M} 87\left(5^{\prime} \phi\right)$ by SIS 
The image processing can be done by folding the response function, which is rather complicated for extended objects. Raw image of A644 observed in $1-10 \mathrm{keV}$ for $50 \mathrm{ksec}$ by GIS is shown in Fig.3, which is completely covered in the GIS field of view. The surface brightness distribution against the projected radius is fitted by a standard $\beta$-model,

$$
\mathrm{I}(\mathrm{r})=\mathrm{I}(0)\left(1+\left(\mathbf{r} / \mathbf{r}_{\mathrm{c}}\right)^{2}\right)^{-3 \beta+1 / 2} \text {, }
$$

where $r_{c}$ is core radius, assuming spherical symmetric distribution. $r_{c}$ and $\beta$ are obtained to be 3 ' and 0.68 , respectively.

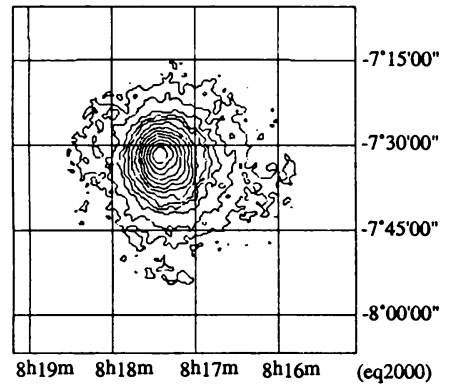

Figure 3. X-ray image of A644 by GIS

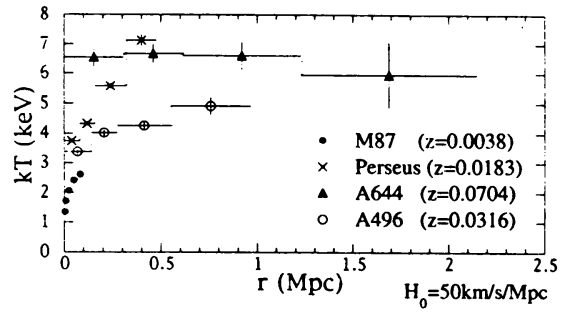

Figure 4. Plasma temperature(kT) vs. projected radius(r)

\section{Cooling flow}

Well relaxed clusters with a central dominant galaxy show an evidence of cooling flow which are observationally recognized as excess emissions and absorption in the central core region and radially decreasing temperature distribution to the cluster center. Spectro-imaging observations with ASCA were clearly revealed the radial gradient of temperatures in many clusters as well as the abundance gradient in some cases. In Fig. 4 the temperature distribution of M87/Vir, A496, the Per cluster and A644 is shown against the projected radius $(\mathrm{Mpc})$ with $\mathrm{H}_{0}=50 \mathrm{~km} / \mathrm{sec} / \mathrm{Mpc}$. Angular radius of these clusters are divided into annular ring of $2.5^{\prime}, 5^{\prime}, 10^{\prime}$ and $15^{\prime}$. Generally speaking, low temperature clusters show steep distribution.

\section{Merging}

Spherically symmetric clusters without a central dominant galaxy classified as nXD seem to be isothermal and in the hydrostatic equilibrium. These clusters have relatively high temperature, low abundance and less central condensation than cooling flow clusters. The temperature of the Coma cluster averaged over whole cluster was obtained to be $8.09 \mathrm{keV}$ by 
Ginga. ASCA observed spatially resolved spectra within the radius of 40'. It shows inhomogeneous temperature distribution in the range of $5.7-12 \mathrm{keV}$. The Coma accompanies a subcluster which shows low temperature[5]. This fact indicates that different temperature component corresponds to different substructure going to merge in a cluster potential.

\section{Determination of the Hubble constant}

Combining the decrement of the brightness temperature of the cosmic microwave background through an intracluster gas ( $\mathrm{S}-\mathrm{Z}$ effect) with plasma temperature obtained by ASCA and the surface brightness profile observed by Einstein, we have derived $\mathrm{H}_{0}$ to be less than $50 \mathrm{~km} / \mathrm{s} / \mathrm{Mpc}$ for $\mathrm{A} 665$ $(\mathrm{z}=0.1816)$ and CL0016+16 $(\mathrm{z}=0.545)[3]$. The Hubble space telescope observations of galaxies in the distance to Coma cluster $(\mathrm{z}=0.0235)$ gave the value to be $80(+/-17) \mathrm{km} / \mathrm{s} / \mathrm{Mpc}[6]$. We have proposed another method by means of resonant scattering effect of $\mathrm{Fe}-\mathrm{K}$ emission lines in an intracluster gas, as $\mathrm{H}_{0}$ is expressed as

$\left.H_{0}=73(\mathrm{~km} / \mathrm{sec} / \mathrm{Mpc})\left(T / 10^{8} \mathrm{~K}\right)^{-1 / 2}(\theta / \mathrm{mrad}) \frac{<\mathrm{n}_{\mathrm{e}}>}{5 \times 10^{-3}} \mathrm{~cm}-3\right) \frac{A b(F e)}{\tau\left(K_{\alpha}\right)}(z / 0.1)$

where $\mathrm{T}$ is plasma temperature, $\theta$ angular size, $\left\langle\mathrm{n}_{\mathrm{e}}\right\rangle$ mean electron density, $\mathrm{Ab}(\mathrm{Fe})$ iron abundance and $\tau\left(\mathrm{K}_{\alpha}\right)$ optical depth of $\mathrm{Fe}-\mathrm{K}_{\alpha}$ emission lines. This method with ASCA data is applicable to spherically symmetric isothermal clusters in $z=0.01-0.1$. We expect that it would be possible to derive the $z$ dependence of $\mathrm{H}_{0}$.

\section{Summary}

Spatially resolved X-ray spectra of clusters of galaxies have been observed in the energy range of $0.5-10 \mathrm{keV}$ and in the redshift range up to 0.5 by ASCA. The cooling flow and merging are directly deduced from the temperature and abundance distribution in an intracluster medium. The determination of the Hubble constant is discussed by means of SunyaevZeldovich effect and resonant scattering effect of $\mathrm{Fe}-\mathrm{K}$ emission lines.

\section{References}

[1] Briel, U.G., and Henry, P.J., 1993, Astr. Astrophys., 278, 379.

[2] Yamashita, K., 1994, Clusters of Galaxies, eds. F. Durret, A. Mazure and J. Tran Thanh Van, Editions Frontiers, p.153.

[3] Tanka, Y., etal., 1994, Publ. Astron. Soc. Japan, 46, L37.

[4] Serlemitsos, P.J., et al., 1994, Publ. Astron. Soc. Japan, 47, 105.

[5] Briel, U.G., et al., 1992, Astr. Astrophys., 259, L31.

[6] Freedman, W.L., et al., 1994, Nature, 371,757. 